\title{
Application of microbial flocculants in sewage treatment
}

\author{
Chenxiao $\mathrm{Xu}^{1, *}$ \\ ${ }^{1}$ College of Resoueces and Environment, Southwest University, Chongqing, China
}

\begin{abstract}
At present, sewage from agriculture, industry and urban life has become the three major sources of sewage today. As global wastewater emissions continue to increase, people are trying to find more effective solutions. Among them, flocculation technology is widely favored as a more economical and simple one among many water treatment technologies. Inorganic and organic polymer flocculants are two types of chemical flocculants developed and applied in China for a long time. Both types of flocculants have their own advantages in different fields. However, in the process of using these two types of sewage treatment technology, it will bring many adverse effects on human health and ecological environment. Inorganic flocculants have a fast flocculation speed, and have a high specific gravity of alum flowers, and they are easy to sink, but they will bring a large amount of inorganic ions such as aluminum and iron into the treated liquid, and excessive intake of aluminum ions by the human body may cause dementia A series of diseases. Strong corrosiveness is the biggest problem of iron salt flocculants in use. It will shorten the service life of the equipment and bring color to the treated water. Organic polymer flocculant flocculates faster than inorganic flocculant, and has more advantages in terms of dosage, separation process, adaptability, etc., because some polymer flocculants monomer itself is toxic, even its hydrolysis or degradation the product is also toxic (such as polyacrylamide monomer). Therefore, researchers are committed to finding efficient, safe, and nonpolluting flocculants to meet the requirements for safe use of flocculants and the reduction of secondary pollution during wastewater treatment. Compared with chemical flocculants, the outstanding advantages have caused the rapid development of microbial flocculants, and its outstanding advantages without secondary pollution have attracted the attention of many researchers, and the development prospect is broad.
\end{abstract}

\section{Features of polymer microbial flocculant}

The flocculant can cause suspended solid particles in the liquid to aggregate and solidify so as to achieve the purpose of sinking. The new type of microbial flocculant is a natural high-molecular organic substance, which has the following characteristics:

\subsection{Safe and non-toxic.}

After a series of tests, it was concluded that microbial flocculants can be used in the pharmaceutical and food industries, and that the waste water treated with microbial flocculants can be recycled and reused, and the emissions can be effectively controlled., In the pharmaceutical and food industry wastewater treatment links play an important role.

\subsection{High efficiency.}

Using the same amount of flocculant, the new polymer microbial flocculant is more efficient than the conventional flocculant.

\subsection{No secondary pollution.}

Microbial flocculants can be produced by different types of microorganisms, and their components are also different, which is more complicated. Nowadays, most flocculants are made of high-molecular substances such as cellulose and glycoprotein and have relatively strong degradation ability. Therefore, the use of new-type microbial flocculants will not cause secondary pollution.

\subsection{Widely used.}

It has a good effect on the treatment of dye wastewater, building material wastewater, and livestock wastewater.

\section{Flocculation mechanism of microbial flocculants}

After the flocculant is added to the water, the repulsive energy between the particles is reduced mainly by the compression of the electric double layer, the neutralization of the charge, the adsorption bridging effect and the net capture style, and finally the aggregation and flocculation occur. Microbial flocculants are charged 
biological macromolecules, and their flocculant mechanisms include the following:

\subsection{Bridge action mechanism}

When the concentration of flocculant biomacromolecules is low, long biomolecule chains adsorbed on the surface of one particle may be adsorbed on the surface of another particle at the same time. Flocculation.

\subsection{Charge neutralization mechanism}

The surface of colloidal particles is generally negatively charged. When a chain-like macromolecular flocculant or its hydrolysate with a positive charge is near the surface of the colloidal particles or adsorbed on the surface of the colloidal particles, it will neutralize the colloid A part of the negative charge on the surface of the particles reduces the electrostatic repulsive force, so that colloid particles can collide and aggregate.

\subsection{Rolling effect}

When the amount of microbial flocculant is fixed and small floc is formed, it can quickly catch nets under the action of gravity and sweep the colloidal particles in the water to produce precipitation and separation, which is called roll sweep or net catch. This effect is basically a mechanical effect, and the amount of flocculation required is inversely proportional to the amount of impurities in the raw water. When the content of raw hydrocolloids is small, the required flocculation dose is large, and vice versa.

\section{Application mechanism of flocculation in wastewater treatment}

During the flocculation process, the surface of the colloidal particles is charged. Because the unit volume and surface area of the particle size are relatively large, the surface energy is also large, so that it can adsorb polar molecules or ions in water. At the same time, the molecules on the surface of the colloidal particles can ionize with the groups, so that they can chemically react with water molecules, so that new compounds can be generated, and this compound can ionize ions. This adsorbs a layer of ions with the same charge on the surface of the colloid, forming an electric double layer structure. Because of the electrostatic attraction, many ions with opposite signs will be attracted in the solution. These ions with opposite signs can diffuse into water. The closer to the surface of the gum core, the higher the concentration of the ion. On the contrary, the ion concentration will be lower as the distance from the surface of the gum core is lower. The positive and negative ions are not neatly arranged on a surface, and are in the form of diffusion in water, and then a diffused electric double layer structure is formed, and a relatively strong layer of substance is formed on the surface of the gum core. This layer of substance is called an adsorption layer. This adsorption layer will form a micelle-fixed layer with potential ions.
When the colloid moves effectively, the colloid will move along with it, so the particles on the periphery of the diffusion layer will be thrown away during the entire movement, Electricity generated on the surface of the moving rubber particles will have a certain potential difference. During the movement of colloids, they are charged particles. If they are close to each other, colloids with the same charge cannot be aggregated due to the interaction between the particles. Therefore, during the movement of colloids, the colloids cannot polymerize with each other [1].

Table 1. The test setting of influence of $\mathrm{Fe} 2+$ 和 $\mathrm{Fe} 3+$ on the key component EPS of MBF

\begin{tabular}{|c|c|c|c|c|c|c|c|}
\hline & $\mathrm{R} 1$ & $\mathrm{R} 2$ & $\mathrm{R} 3$ & $\mathrm{R} 4$ & $\mathrm{R} 5$ & $\mathrm{R} 6$ & $\mathrm{R} 7$ \\
\hline $\begin{array}{c}\mathrm{Fe} 2+ \\
(\mathrm{mg} / \mathrm{L})\end{array}$ & 0 & 6 & 12 & 18 & 0 & 0 & 0 \\
\hline $\begin{array}{c}\mathrm{Fe} 3+ \\
(\mathrm{mg} / \mathrm{L})\end{array}$ & 0 & 0 & 0 & 0 & 6 & 12 & 18 \\
\hline
\end{tabular}

\section{Application of microbial flocculants in wastewater treatment}

Treatment of industrial wastewater: Generally, the processing methods of coal preparation process will produce wastewater such as slime water, that is, industrial wastewater. However, the effective treatment of highmolecular microbial flocculants will produce good results. In addition, in terms of price, high-molecular flocculants are cheap, do not cause secondary pollution to the environment, and can effectively degrade organic pollutants. The study of microbial flocculant and its effect on slime water flocculation in foreign countries are earlier than those in China. As early as the 1960s, foreign countries have studied the results of flocculation treatment of wastewater by Mycobacterium phlei, which has obvious benefits. In China, Liu Zhiyong was the first person to investigate and investigate the flocculant of slime wastewater. After testing, he obtained microbial flocculants produced by primitive, ultraviolet, domestication and chemical mutagenesis, which produced various bacteria on the treatment of slime wastewater. obvious.

\section{(1) Treatment of domestic wastewater}

A kind of mixed and efficient flora can be separated from urban domestic wastewater. The function of this flora is mainly to degrade and flocculate domestic wastewater, which can make the five-day biochemical oxygen demand (BOD5) and chemical oxygen demand (COD) in sewage. Was largely removed. Using polymer microbial flocculants to effectively treat domestic sewage and wastewater, the removal rate of chemical oxygen demand can reach $68 \%$, and the removal rate of suspended matter can reach $91 \%$. 


\section{(2) Treatment of wastewater from food processing} industry

For the treatment of wastewater from food processing plants, high molecular microbial flocculants can be used, which can achieve the desired effect. The wastewater from the production of monosodium glutamate can be treated with pullulan, a polymer microbial flocculant. The removal rate of suspended solids, chemical oxygen demand and suspended solids can reach $40 \%$, and the turbidity removal rate can reach $99 \%$. Some people use strain A-9 when treating starch wastewater. The suspended solids removal rate can reach $85.5 \%$, and the chemical oxygen demand removal rate can reach $68.5 \%$. The treatment effect is more obvious than ordinary chemical flocculants, and it can the protein component is recovered and made into feed.

\section{(3) Treatment of printing and dyeing wastewater}

The current wastewater treatment technology makes the five-day biochemical oxygen demand index lower, and the discoloration effect of the soluble pigment solution is not obvious, but the soluble pigment can be treated by using a microbial flocculant to achieve the expected effect.

\section{(4) Treatment of fermented products}

The use of microbial flocculants has good sedimentation properties for biological cells, which can remove the bacteria in the fermentation broth, which can greatly reduce energy consumption and production costs, and is easy to manage and simple to use. In the brewing industry, the use of yeast with better flocculation performance to replace the yeast with poor or no flocculation performance can produce beer of good quality; in addition, it is also widely used in the production of bread fermentation yeast and bioethanol. A kind of microbial flocculant, which can achieve good results, remove solids, reduce product processing time, help to degrade unstable substances and save costs.

\section{Development Trend of Polymeric Microbial Flocculants}

In a variety of wastewater treatment, the use of microbial flocculants can mostly achieve the previously expected results. Therefore, in the future development, we can take the research and development of microbial flocculants as a focus of development. The first step is to establish and improve the method for selecting flocculants. At present, the initial and re-screening of flocculants are carried out using kaolin suspension, which has certain errors and low efficiency. Therefore, in order to improve the work efficiency, quickly find an effective way to screen the flocculant-producing bacteria Is a key in development. Secondly, it is necessary to organize researchers to conduct a comprehensive and in-depth study on the flocculation mechanism of microbial flocculants. The research on the flocculation mechanism of high-molecular microbial flocculants should not be carried out only from a biological perspective, but also from multiple perspectives and chemistry aspects such as chemical and physical aspects [2]. According to the existing flocculation mechanism, different types of wastewater treatment Effect and role, explore its law, conduct targeted research on different water quality, develop different types of microbial flocculants, and achieve the right medicine. This can achieve the purpose of reducing the amount of flocculant use while achieving significant results, and further make the treatment of wastewater Costs are minimized. Therefore, the research on the flocculation mechanism can fundamentally achieve the expected wastewater treatment effect. Finally, we need to build engineered bacteria. If the production of flocculants mainly depends on the natural environment, then the realization of normalized and large-scale production and use is very difficult, so high-tech biotechnology can be used to construct engineering bacteria. During the construction process, the flocculation gene can be obtained by molecular biology technology, and then the gene is mutated to breed the bacteria, and finally a bioengineered bacteria with flocculation function is produced [3].

\section{Conclusion}

The research and development and application of microbial flocculants are constantly expanding to highefficiency, new-type, and cheap. On the one hand, it is derived from people's increasing attention to ecological construction and environmental protection. The living environment has become better, also because it is a longlasting project of fukuzawa contemporary and beneficial to the future [4]. The research of microbial flocculants and the continuous application in wastewater treatment have not only continued to expand the field of use of water treatment technology, but also set the wastewater treatment technology to a new level. But at present, there are still a series of problems in the development of microbial flocculants, such as: the cost is too high to meet the needs of industrial production $\downarrow$ most of the research is still in the screening stage of bacteria, etc., but its development will not Problem and stop. The selection of excellent strains and the continuous integration of biotechnology and various water treatment technologies provide effective solutions for many problems that arise during the research of microbial flocculants. In the long run, microbial flocculants will become one of the hot topics in the future [5].

\section{References}

[1] Xiong Xuewen, Zhang Hongwei. Talking about the application of microbial flocculants in sewage treatment [J]. Jiangxi Building Materials, 2014 (19).

[2] Li Nan. Microbial flocculant and its application in sewage treatment [J]. Earth, 2015 (08).

[3] Zhu Bin, Wu Zhiqin. Application of microbial flocculants in wastewater treatment [J]. Journal of Zunyi Normal University, 2013 (05). 
[4] Ji Liang, Xiang Bo, Li Qianqian, Li Yijiu(Department of Chemistry, Tongji University, Shanghai 200092,China); Research on chelating properties of modified corn starch for heavy metals[J];Industrial Water Treatment;2006-12

[5] Tao Tao et al; Study on Treatment of Concentrated Wastewater of Monosodium Glutamate Production[J]; Water \& Wastewater Engineering; 2001-01 